\title{
La « grammaire nationale » de Damourette et Pichon : idiomologie et idéologie
}

\author{
Anne-Gaëlle Toutain ${ }^{11}$ \\ ${ }^{1}$ Institut de langue et de littérature françaises de l’Université de Berne/Laboratoire « Histoire des \\ théories linguistiques » (UMR7597, Paris).
}

\begin{abstract}
Résumé. Nous nous efforçons, dans cet article, de faire paraître le rôle joué par l'obstacle épistémologique de l'idiome dans la "grammaire nationale » de Damourette et Pichon. Nous montrons ainsi successivement la dépendance de la définition tout à la fois raciale et linguistique qu'ils donnent de la nation à l'égard d'une représentation de la langue en termes d'entité, et l'inscription de leur appréhension de la langue comme «pensée-langage » dans la problématique des rapports son/sens qui est corrélative de la définition traditionnelle du signe. Cette critique de l'élaboration linguistique de Damourette et Pichon est rendue possible par la comparaison avec l'élaboration saussurienne. Elle vise par ailleurs à faire paraître le caractère protéiforme de l'obstacle épistémologique de l'idiome, dont les enjeux sont cruciaux pour la linguistique et les sciences du langage contemporaines.
\end{abstract}

\begin{abstract}
Damourette and Pichon's «national grammar»: idiomology and ideology. In this paper we aim at highlighting the role played by the epistemological obstacle (obstacle épistémologique) of the idiom in the Damourette and Pichon's «national grammar». We thus show, first, that the racial and linguistic definition that they give for the nation depends on a representation of language as an entity. Second, we demonstrate that their conception of language as «thought-language" («pensée-langage ») is internal to sound-meaning relationship problematics, which is a correlate of the traditional definition of the sign. This critical examination of Damourette and Pichon's linguistic work is made possible by the comparison with Saussurean linguistics. Furthermore, its aim is to highlight the proteiform character of the epistemological obstacle of the idiom, whose stakes are crucial for contemporary linguistics and language sciences.
\end{abstract}

«Pour faire la grammaire française que nous concevons, il fallait donc être Français. Nous le sommes. Il serait à souhaiter, selon nous, que des nationaux des différents pays s'efforçassent de construire, suivant la même méthode, les grammaires respectives de leurs idiomes. » (1, tome I : p. 15), écrivent Damourette et Pichon au début du deuxième chapitre de Des mots à la pensée. Essai de grammaire de la langue française (1911-1927) [1]. On

${ }^{1}$ Corresponding author : annegaelletoutain@yahoo.fr 
peut, à cet égard, à la suite de Valelia Muni Toke [2], et de Damourette et Pichon euxmêmes ${ }^{1}$, parler, à propos de l'entreprise de ces deux linguistes, de " grammaire nationale " ou de « linguistique nationale $»^{2}$. Il ne s'agit pas seulement, en effet, d'être locuteur natif du français, mais il faut en outre être membre de la «nation » française, et l'arrière-plan d'une telle proposition est double. En premier lieu, selon Damourette et Pichon, « le langage est de la pensée » (1, tome I : p. 11), et «l'unité réelle du groupe formé par ceux qui parlent la même langue consiste en ce que leur pensée, au moins dans ce domaine supérieur que le langage seul peut atteindre, est guidée, d'ailleurs en grande partie inconsciemment, par un même système intellectuel : le système taxiématique ${ }^{3}$ propre ou grammaire propre de leur langue » $(1$, tome I : p. 14). Or, dans cette perspective, «[i]l faut [...], pour qu'un esprit saisisse réellement tout le contenu sémantique d'un discours, qu'il ait, dès l'infantile époque de sa formation, été modelé selon le système taxiématique de l'idiome dans lequel la pensée de son interlocuteur s'exprime » $(1$, tome I : p. 14), et «[t]elle est la véritable raison de ce fait bien connu qu'il est absolûment impossible de jamais comprendre parfaitement un idiome autre que le sien propre » (1, tome I : p. 14). Dès lors, dans la mesure où «la tâche du grammairien [est] d'amener en entier dans le champ de la conscience le système taxiématique qui baigne en grande partie dans l'inconscient » (1, tome I : p. 15), il est «à peu près indispensable que le grammairien possède en lui-même les éléments inconscients sur lesquels il va projeter la lumière » (1, tome I : p. 15). Cette conception des rapports entre langage et pensée se double cependant, en second lieu, de propositions d'ordre «biologique $»$, relatives aux rapports entre « race» et langage, la question de la race étant de ce fait même impliquée dans celle de la nation. Dans ce même deuxième chapitre de Des mots à la pensée. Essai de grammaire de la langue française, Damourette et Pichon posent en effet la question : "Y a-t-il un rapport entre le parler d'un groupement humain et sa race, c'est-à-dire l'ensemble des caractères qu'il possède en commun et par hérédité ?» (1, tome I : p. 17). Selon eux, il existe quelque chose de tel qu'une « hérédité mentale » $(1$, tome I : p. 17), et celle-ci joue un rôle dans la formation du parler d'un individu, déterminant notamment, lors de l'apprentissage comme langue maternelle d'une langue étrangère, «d'imperceptibles déformations » (1, tome I : p. 17) ; de même, « toute race qui adopte le parler d'une autre race le modifie profondément dans sa phonétique et sa syntaxe, conformément à ses organes et à son esprit propres » $(1$, tome I : p. 18). Se trouve ensuite défini le terme de nation:

\begin{abstract}
$[\ldots]$ on devra penser que les hommes parlant la même langue (au sens que nous accordons à ce mot) sont sensiblement de même race ; ils ont les mêmes traditions, les mêmes conceptions sémantiques, la même littérature, la même science. C'est ce groupe, et ce groupe seul, qui mérite, par conséquent, le nom de nation et la nation ainsi définie représente bien l'unité d'où doit partir la classification la moins arbitraire du genre humain. L'importance extrême de la nationalité ainsi comprise, importance qui repose sur une foule de faits non point imaginaires et muables, mais réels et constants, en fait, dans les grands pays de l'Europe occidentale, le principal support de l'idée de patrie dans ce qu'elle peut avoir de plus noble et de plus solide 5 . (1, tome I : p. 22).
\end{abstract}

La linguistique de Damourette et Pichon apparaît ainsi inséparable d'un arrière-plan idéologique, le nationalisme, nationalisme non exempt d'antisémitisme et de racisme. On sait que leurs auteurs étaient maurrassiens. Il importe cependant de noter que Damourette et Pichon n'ont pas l'exclusivité de ce type de propos. S'ils parlent ainsi, entre autres, dans Des mots à la pensée. Essai de grammaire de la langue française, du grec et du français comme de langues «ayant été parlées par les deux plus affinés des peuples historiquement connus » (1, tome I : p. 16), de sorte qu' «elles sont en possession des systèmes taxiématiques les plus riches et les plus fins » (1, tome I : p. 16), ou évoquent, dans ce 
même ouvrage, un « état mal dégrossi de la pensée, que, dès leur aurore, les langues indoeuropéennes semblent avoir déjà dépassé » (1, tome I : p. 280), mais qui « paraît pourtant être celui de bien des langues parlées de nos jours » (1, tome I : p. 280), comme le chinois, il est de même question, par exemple, dans les Leçons de linguiistique 1946-1947 C de Gustave Guillaume, de «nos langues évoluées » $(3:$ p. 11), et si le chinois est à l'inverse considéré dans cet ouvrage comme "le résultat d'une civilisation spirituelle très développée » (3:p. 113), Guillaume poursuit :

Toutes les langues à caractères sont fort loin, du reste, à cet égard, de s'égaler au chinois; et il en est un assez grand nombre qui ne permettent pas la saisie abstraite des éléments formateurs employés, la saisie de ceux-ci ne pouvant avoir lieu que dans le cadre d'un acte de langage engagé.

$[\ldots]$

Les langues en question, qui comportent, on le conçoit, une foule de modalités constructives, difficiles du reste à bien situer les unes par rapport aux autres, sont les plus primitives de toutes. Elles nous font remonter, par leur primitivité, à l'époque où la formation de la langue consistait à identifier, dans un discours tenté, essayé, les moyens constants et réguliers de sa formation. Par ces langues on est ramené à l'état de moindre séparation de la langue et du discours, la langue ne s'étant pas encore bien retirée de celui-ci. ( $3:$ p. 113).

Or, un point commun des élaborations théoriques de Damourette et Pichon et de Guillaume est leur caractère de linguistique "psychologique », c'est-à-dire dans le cadre de laquelle le langage apparaît comme le lieu de la pensée, dont il est aussi bien la condition de possibilité que la manifestation privilégiée et le produit. Ce caractère est en réalité le fruit d'un mode particulier de représentation du rapport son/sens constitutif du langage, ainsi que nous nous proposons de le faire apparaître dans cet article, où nous nous attacherons à montrer que le caractère «national» de la linguistique de Damourette et Pichon, bien qu'idéologiquement déterminé, est par ailleurs, d'un point de vue épistémologique, une conséquence de l'obstacle épistémologique de l'idiome, autrement dit, que derrière l'idéologie - dont il ne s'agit certes pas de nier l'existence - se profile une idéologie scientifique, au sens de Georges Canguilhem [dans 4]. Nous verrons ainsi comment la définition citée de la nation se fonde sur une représentation de la langue en termes d'entité (1), puis la manière dont Damourette et Pichon reprennent à leur compte la conception commune du rapport son/sens, et sont ainsi conduits au postulat d'un objet conçu comme le lieu d'une articulation entre linguistique et psychanalyse, mais dont la caractéristique majeure est au contraire de n'être ni linguistique, ni psychanalytique (2).

\section{Idiome et entité}

La définition de la nation que nous avons citée dans ce qui précède caractérise celle-ci comme un groupe dont l'unité est doublement déterminée, comme unité de race et comme unité linguistique. Cette définition repose bien entendu avant tout sur le présupposé de l'existence de quelque chose de tel que des races humaines, que l'on sait aujourd'hui, avec le développement de la génétique, scientifiquement infondé. C'est ainsi que pour Damourette et Pichon, si, comme nous l'avons vu ci-dessus, la race n'est pas sans effet sur la langue, inversement, et pour cette raison même, l'étude linguistique peut apporter des informations sur les rapports, notamment raciaux, entre les peuples. Ils affirment en effet, à la suite du développement cité alors ${ }^{6}$ :

En somme, contrairement aux idées des comparatistes de l'époque héroïque, la correspondance phonétique de deux idiomes et leur possible explication par un même parler antérieur n'implique pas du 
tout que les peuples parlant ces deux idiomes soient de races apparentées. Mais le fait qu'ils aient divergé à différents points de vue, et principalement au point de vue taxiématique, prouve au contraire que les peuples les parlant avaient une hérédité mentale notablement différente.

D'autre part, des idiomes n'appartenant pas aux mêmes cases de la classification linguistique matérielle pourront avoir dans leur système taxiématique des ressemblances telles qu'elles dénonceront un apparentement réel entre les peuples parlant ces idiomes. (1, tome I : p. 18).

Ce deuxième chapitre de Des mots à la pensée. Essai de grammaire de la langue française, intitulé "La constitution extérieure de la langue française », donne de même notamment à lire ${ }^{7}$ une sommaire histoire linguistique de la France dont l'un des objectifs est de montrer que les diverses invasions n'ont modifié que de manière marginale « le sang des habitants de la Gaule » (1, tome I : p. 25), de sorte que finalement « il semble bien qu'on puisse en conclure légitimement à l'existence d'une race française au moins pour autant que la notion de race est liée à celle de parenté » (1, tome I : p. 27), fait d'importance puisque l'influence de la race sur la langue doit se vérifier dans « [1]'influence des Gaulois sur la constitution du français » (1, tome I : p. 29), influence dont Damourette et Pichon établissent le caractère « indéniable » $(1$, tome $\mathrm{I}:$ p. 29$)$.

Cependant, et c'est ce que nous voudrions mettre en évidence ici, ce présupposé (et les références «scientifiques » vieillies et choisies qui lui sont attachées) se double d'une représentation spontanée - au sens bachelardien - en termes d'idiomes, ces derniers étant conçus comme des entités délimitées. Damourette et Pichon envisagent ainsi également dans ce deuxième chapitre de leur Essai, et précisément à la suite des développements relatifs au rapport entre langue et race, le "problème [...] du morcellement d'un parler originel en plusieurs parlers dérivés » $(1 \text {, tome } \mathrm{I}: \text { p. } 18)^{8}$. De nouveau, la notion de race vaut principe explicatif puisque l'unité linguistique des Sémites peut être considérée comme " une preuve accessoire de la preuve anthropologique de leur unité de sang » (1, tome I : p. 18) et que «si un idiome continue à être parlé par les descendants de ceux qui l'ont créé, les différences ne deviennent jamais très profondes » (1, tome I : p. 19). Il s'agit néanmoins de rendre compte d'un "morcellement », c'est-à-dire de la naissance d'idiomes distincts, définitoires de «nations », à partir d'un autre idiome, tout aussi distinct et national, que Damourette et Pichon appellent « l'idiome originel» (1, tome I : p. 19) et dont ils affirment ainsi qu'il était "parlé par des hommes étroitement apparentés » (1, tome I : p. 19). On retrouve alors les notions traditionnelles - et communes, au sens de la connaissance commune que viennent rectifier les connaissances scientifiques - de dialecte et de patois, auxquelles Damourette et Pichon confèrent seulement un fondement racial. Ces derniers distinguent dans la suite de ce développement entre «deux ordres de différences entre les parlers dérivés d'un même idiome» (1, tome I : p. 19), les «différences dialectales » (1, tome I : p. 19) qui séparent les dialectes d'une même « lingualité », auxquels l'apparentement des hommes qui les parlent confère une unité syntaxique, grammaticale et phonétique, de sorte que ces différences dialectales demeurent minimes, et les «divergences idiomatiques » (1, tome I : p. 19) séparant les idiomes de lingualités différentes, et « qui procèdent de l'origine ethnique différente des peuples ayant parlé l'idiome originel» (1, tome I : p. 19). Les patois proviennent pour leur part de l'établissement, au sein d'une lingualité, de la domination d'un dialecte, dont les autres deviennent ainsi des patois ${ }^{9}$. Ces patois sont considérés, comme ils le sont communément, comme inférieurs à la «langue » que constitue le dialecte dominateur ${ }^{10}$, et leur disparition au profit de cette dernière consacre le triomphe de la patrie ${ }^{11}$. Intervient ainsi, à cet endroit, un deuxième facteur explicatif des différences entre les langues, s'ajoutant à celui des communautés ou différences raciales : les circonstances historiques, qui seront de nouveau invoquées plus loin pour expliquer la division entre langues d'oïl et langues d'oc : 


\begin{abstract}
D'après ce que nous avons dit au $\S 18$, la race des Français du sud diffère bien peu de celle des Français du nord. Ce n'est donc pas dans un facteur de race proprement dite qu'il faut chercher l'explication de la différence si nette entre les parlers d'oc et ceux d'oui. C'est bien plutôt dans des circonstances historiques. (1, tome I : p. 33).
\end{abstract}

Le fait remarquable est que ces deux principes explicatifs - racial et historique - sont dotés d'un statut analogue, étant tous deux invoqués pour rendre compte d'unités et de différences dont le postulat est premier. Le développement relatif à cette division est d'ailleurs extrêmement significatif pour notre propos puisqu'y sont mentionnées certaines propositions de Gaston Paris [5], ainsi que la Wellentheorie de Johannes Schmidt [6], que Damourette et Pichon écartent au nom de l'évidence de l'idiome. Damourette et Pichon posent ainsi que « [c]ertainement le latin avait abouti dans chacun des points de la Gaule romaine à un dialecte spécial, et tous ces dialectes formèrent et forment encore aujourd'hui, en tant que patois, un dégradé linguistique à peu près continu, ou du moins dans lequel les limites des différents phénomènes de détail ne se recouvrent pas » $(1$, tome $\mathrm{I}: \mathrm{p}$. 30). C'est ce dont Gaston Paris, qu'ils citent alors, tirait argument pour conclure que la division entre langue d'oc et langue d'oïl est imaginaire, et n'existe que du point de vue littéraire. Damourette et Pichon affirment cependant pour leur part «préfér[er] conserver la division du domaine gallo-roman en deux lingualités principales : la lingualité d'oui ou lingualité francimande et la lingualité d'oc ou lingualité occitaine » (1, tome I : p. 31), invoquant le fait que ce continuum pourrait être étendu aux lingualités voisines, et même à l'ensemble des langues indo-européennes, mais qu'il serait abusif de conclure de ce constat, qui a donné lieu à la Wellentheorie de Schmidt, à l'inexistence de lingualités distinctes :

\begin{abstract}
[...] elle [cette constatation] ne nous paraît aucunement justifier l'abandon de la notion de lingualité. En effet, toute notion scientifique est forcément schématique, puisqu'il n'y a de science que du général, et qu'en matière de sciences expérimentales, on ne peut ranger les faits dans des groupes généraux qu'à condition de les rattacher à des types schématiques. De ce qu'il n'y a aucun symptôme particulier qui soit commun à rigoureusement tous les cas de fièvre typhoïde, doit-on en conclure que la fièvre typhoïde n'est pas une entité clinique ? De ce que les biologistes ne se sont pas mis d'accord sur un caractère diacritique permettant de classer à coup sûr un être inférieur dans le règne animal ou dans le règne végétal, doit-on en conclure que la notion même de règne biologique doive être abandonnée? Évidemment non. (1, tome I : p. 31-32).
\end{abstract}

Quant aux faits positifs convoqués ensuite, ils ne sont pas réellement d'ordre linguistique ; l'un est le fait que «[d]epuis le moyen âge, le pays d'Oc et le pays d'Oui ont eu conscience de faire, jusqu'à un certain point, contraste l'un avec l'autre » (1, tome I : p. 32) ; l'autre est qu' « il semble légitime de ne pas considérer comme de même lingualité des parlers tels que ceux qui s'en servent ne se comprennent point les uns les autres » (1, tome I : p. 32). Il faut noter que dans cette argumentation la représentation des idiomes en termes d'entités délimitées joue le rôle de point de référence. C'est au regard d'une telle représentation, en effet, que les «lingualités» apparaissent comme les seuls «types schématiques » généraux possibles en matière de science linguistique; si la fièvre typhoïde a une étiologie bien caractérisée à l'époque de la rédaction de l'Essai de grammaire de la langue française, il n'en va pas de même des langues, dont la grammaire historique et comparée, puis la dialectologie, avec les remaniements qu'elle impose à cette dernière, bousculent la représentation. Celles-ci conduisent en particulier, comme l'a compris Saussure, à renoncer à cette représentation en termes d'entité. Cette présupposition d'une unité apparaît de nouveau de manière très nette dans le troisième chapitre de l'Essai de grammaire de la langue française, intitulé «L'organisation interne de la langue 
française $»^{12}$, où Damourette et Pichon s'attachent à définir, distinguer et caractériser des « usances », « disances », « parlures » et « jargons », mais pour conclure que « la diversité des habitudes des différents Français n'altère en rien l'unité foncière de la langue » (1, tome I : p. 53) ${ }^{13}$.

C'est donc au nom de l'évidence de l'idiome que Damourette et Pichon écartent les propositions de Paris et de Schmidt, que Saussure invoquait pour sa part afin d'en énoncer les conséquences théoriques : à savoir la nécessité d'une rupture avec cette représentation de la langue en termes d'entité. Saussure s'attachait en effet pour sa part à un tout autre type de révision de la doctrine des «comparatistes de l'époque héroïque » (1, tome I : p. 18), pour reprendre une expression de Damourette et Pichon citée ci-dessus. Dès les conférences de 1891 [dans 7], Saussure renonce, en se référant aux travaux de Paul Meyer, à toute délimitation de la langue dans l'espace, affirmant que les notions de dialecte et de langue sont purement conventionnelles, comme telles et dans leur différence, dans la mesure où l'observation empirique ne nous révèle que des caractères dialectaux et un continuum linguistique ininterrompu ${ }^{14}$. Dans les trois cours, il s'attache à une critique de la «théorie des essaims » ou "théorie des migrations », corrélative d'une représentation en termes de Stammbaum, et selon laquelle toute apparition d'une langue nouvelle est le résultat d'une migration, la séparation géographique étant à l'origine de cette différenciation $^{15}$. Le développement du troisième cours est le plus remarquable. Saussure y insiste tout d'abord sur le caractère inaugural du constat de la diversité des idiomes, auquel on doit précisément le terme même d'idiome, qui désigne « la langue considérée dans ses caractères spéciaux, dans les caractères qui sont propres à un peuple » $(10:$ p. 95). Le deuxième constat est celui de l'existence de ressemblances entre les langues, qui conduit à la grammaire comparée et implique la distinction de deux types de diversité : entre langues d'une même famille, la «diversité dans la parenté » (10: p. 98), et entre langues non apparentées. Selon le deuxième cours, cette «diversité hors de toute parenté reconnaissable » $(10:$ p. 98$)$ pose la question de la race :

Diversité radicale : <par exemple> entre les langues indo-européennes et le chinois. Les bases d'expression de la pensée diffèrent entre ces langues. La question de race se pose et nous voyons poindre des rapports avec l'ethnologie. » $(9:$ p. 4$)$.

Cette question est de nouveau évoquée dans le troisième cours, pour être laissée en suspens, comme complexe et, comme telle, non linguistique. Saussure affirme en particulier $^{16}$ :

Autre observation: Si la langue se donne tout de suite comme une chose géographiquement diverse, ne doit-on pas la considérer comme ethniquement diverse ? Cette question est très complexe. L'idée de race a cette différence constatée dans le parler. Sans doute on pourrait aller au-delà de la diversité géographique, mais les rapports entre la langue et l'ethnisme sont beaucoup plus complexes. Dès qu'il est question de la langue comme caractère de race, nous faisons intervenir le principe de variation dans le temps, ou de relative résistance à la variation dans le temps; ce n'est que par la persistance de la langue qu'elle peut être plus ou moins un caractère de race.

Déjà à cause de cela on mêlerait des considérations qui ne sont pas immédiatement offertes. (10: p. 96-97).

Il est significatif, à cet égard, que la notion de « diversité radicale » soit définie, dans ce troisième cours, comme une diversité « hors de toute parenté », c'est-à-dire comme une diversité échappant à la prise que donne, à l'époque de Saussure, la linguistique, et qui est avant tout celle de la grammaire historique et comparée. Il apparaît en effet que la démarche saussurienne est une démarche d'élaboration linguistique du donné ; si le constat 
initial est celui de la diversité des langues, il s'agit à présent de rendre compte de celle-ci, par une démarche spécifiquement linguistique. Saussure affirme ainsi ensuite :

\begin{abstract}
Nous avons vu que ce fait de la diversité géographique était celui qui frappe tout d'abord. Comment les choses se passent-elles pour que ce fait se produise? Si nous nous plaçons $\left\langle\mathrm{I}^{\circ}\right)>$ devant la diversité absolue, nous sommes en face d'un problème : l'irréductibilité des langues du globe. C'est un problème qui est placé sur le terrain spéculatif, qui nous transporte dans des périodes inaccessibles et qui touche à d'autres questions : l'origine du langage. Nous laisserons ce problème de côté.

Il en est autrement $\left\langle\mathrm{II}^{\circ}\right)>$ de la diversité dans la parenté. Nous sommes placés ici sur le terrain de l'observation. Il nous est donné d'assister à la production de cette diversité et nous pourrons donner des résultats certains. $(10:$ p. 106).
\end{abstract}

C'est donc l'étiologie de cette diversité qui intéresse avant tout Saussure, au lieu que Damourette et Pichon en font le cadre de leur élaboration. La rupture avec la représentation de première venue en termes d'idiomes distincts - ce constat inaugural de la linguistique apparaît de manière très nette dans l'exposé de cette étiologie, tout particulièrement remarquable par son caractère pédagogique. Saussure y critique la théorie des migrations, mais en commençant par le cas de la discontinuité géographique, qui paraît, au premier abord, le plus simple, et en montrant ensuite, pas à pas, que « la discontinuité ne crée pas un cas théorique vraiment important » $(10:$ p. 107). Le changement est en effet dû au seul facteur temps, que l'espace ne fait que "multiplier ${ }^{17}$ » en autorisant des évolutions divergentes. Or, la séparation géographique n'est pas nécessaire à cette divergence, qui conduit au «fractionnement sur place» dont rend compte la Wellentheorie de Johannes Schmidt et qui implique l'abandon de toute représentation en termes d'entité. L'existence d'idiomes distincts, par exemple les langues nationales, est due pour sa part à d'autres types de phénomènes, non linguistiques, comme des déplacements de population ou la formation et l'imposition de langues littéraires ou officielles.

La représentation en termes d'entité apparaît ainsi doublement rectifiée par ce développement du troisième cours : comme représentation préalable, dont la linguistique s'attache à établir l'étiologie, et comme obstacle épistémologique, dont la théorie des migrations témoigne exemplairement ${ }^{18}$, de sorte qu'à cet égard, le principe explicatif de la race mobilisé par Damourette et Pichon apparaît comme une autre forme de celle-ci. Si l'on retrouve en effet chez Saussure deux principes d'explication, diachronique et historique, répondant apparemment à ceux de la race et des circonstances historiques de Damourette et Pichon, les deux élaborations n'ont en réalité rien de commun: les deux principes saussuriens se trouvent hiérarchisés, s'opposant, notamment, comme linguistique et non linguistique, et par ailleurs, déconstruisent, pour en constituer l'étiologie, une représentation de première venue que leurs homologues chez Damourette et Pichon viennent au contraire renforcer dans la mesure même où le premier la redouble. Cette parenté entre la représentation des premiers comparatistes et le nationalisme de Damourette et Pichon fait de cette idéologie une idéologie scientifique et montre comment sujet éthique et sujet épistémologique peuvent conjuguer leurs efforts pour faire barrage à toute psychanalyse de la connaissance scientifique.

Cette collaboration ou cette convergence sont également lisibles dans le premier des deux aspects de la grammaire nationale de Damourette et Pichon que nous avons vus en introduction: la construction d'un objet linguistico-psychanalytique. La référence saussurienne à la théorie de Johannes Schmidt implique en effet, dans ses développements ultimes, la construction, pour la langue, d'une extériorité non objectale, extériorité d'une langue définie comme fonctionnement, conformément à la deuxième rupture opérée par la 
théorie saussurienne : avec la problématique des rapports son/sens, dans laquelle s'inscrit en revanche la théorie de Damourette et Pichon.

\section{La question du rapport son/sens : enjeux de la linguistique " psychologique »}

Lorsqu'il expose, dans le troisième cours, la Wellentheorie de Schmidt, Saussure introduit ${ }^{19}$ une distinction entre deux forces opposées agissant dans toute communauté humaine, qu'il appelle «force du clocher» et «intercourse ». La première agit en faveur de la cohésion d'une communauté restreinte, et a donc un effet diversifiant et particularisant ; la seconde est au contraire un principe unifiant puisqu'elle est la force portant les hommes à la communication ; ces deux forces ne sont par ailleurs distinguables que si l'on considère un point donné du territoire; dès que l'on s'intéresse à une surface, elles apparaissent comme le revers l'une de l'autre : l'intercourse unifiant cette surface est aussi bien la force du clocher l'isolant des autres, et inversement. On retrouve dans cette représentation la substitution des caractères dialectaux aux dialectes, dans la mesure où ces forces opèrent pour chaque caractère, dont elles assurent la propagation. S'y articulent par ailleurs les dimensions historique et sociale de la langue : la langue est toujours héritée, et n'existe que dans cette transmission, mais elle est par ailleurs, comme telle, intrinsèquement sociale, force du clocher et intercourse, qui se confondent en dernière analyse, apparaissant ainsi comme les deux axes de sa circulation. Comme il apparaît en particulier dans le deuxième cours $^{20}$, cette circulation est en réalité le mode d'existence de la langue saussurienne, que Saussure qualifie de «vie sémiologique» $(9:$ p. 12), et qui est celui d'une langue à l'extériorité non objectale, mais constitutive. En lieu et place de la délimitation idiomologique, dont, comme nous l'avons vu, Saussure met en évidence le caractère de représentation de première venue, mais que Damourette et Pichon consolident et essentialisent en parlant de «nation », et qui est une délimitation objectale - définissant, pour chaque idiome, un mode d'existence objectal, c'est-à-dire analogue à celui de tout objet, de toute « chose ", au sens commun et banal du terme -, se trouve ainsi définie une extériorité constitutive, qui est tout à la fois - et indissolublement, car corrélativement une conséquence logique du renoncement à la représentation des idiomes en termes d'entité, et une élaboration théorique du constat empirique de l'existence d'idiomes délimités.

La langue de Damourette et Pichon est tout à la fois, et contradictoirement, individuelle et sociale. On lit ainsi notamment dans Des mots à la pensée. Essai de grammaire de la langue française :

[...] le langage est la partie proprement humaine de la pensée des hommes. Il en découle que chaque homme aura en réalité son parler propre, et que c'est seulement par ces procédés d'abstraction généralisatrice sans lesquels il n'y a pas de science possible que l'on pourra, de notre point de vue, concevoir des parlers collectifs. (1, tome I : p. 16).

Damourette et Pichon parlent ici de «procédés d'abstraction généralisatrice sans lesquels il n'y a pas de science possible », mais il importe de remarquer qu'il s'agit là d'abstraction objectale, consistant, comme nous l'avons vu plus haut, à faire de chaque idiome une organisation d'usances, de disances, de parlures et de jargons, et que ce n'est pas là la seule forme d'abstraction possible en linguistique ou en réalité, plus précisément, qu'il s'agit là d'une abstraction « idiomologique », par opposition à « linguistique », c'està-dire fondée sur l'idiome et non constitutive de l'objet de la linguistique, et présupposant ainsi l'objet qu'elle devrait théoriser. La langue saussurienne, pour sa part, se spécifie par sa double dimension individuelle et sociale, qui n'est pas contradictoire, mais au contraire 
élaborée et théoriquement articulée : articulation de la pensée dans la matière phonique, la langue ne saurait avoir d'autre existence qu'individuelle ; dans le même temps, cependant, elle n'existe comme telle que par son caractère social, qui en constitue précisément l'extériorité. On lit ainsi notamment dans le troisième cours :

On peut dire qu'en prenant un individu nous aurons dans le seul exemplaire l'image de ce qu'est la langue dans la masse sociale. $<\mathrm{Si}$ nous pouvions examiner le dépôt des images verbales dans un individu, conservées, placées dans un certain ordre et classement, nous verrions là le lien social qui constitue la langue > $(10:$ p. 217).

Apparaît bien ici l'enjeu de la définition saussurienne de la langue comme un phénomène social : il ne s'agit pas, comme on l'a cru souvent, d'une existence extérieure aux individus, mais d'une extériorité constitutive de la langue ; la langue est un «trésor individuel », un produit de la faculté de langage, mais qui n'existe comme telle que dans la mesure où elle est sociale, où elle est articulation socialement codée entre pensée et phonie. Citons également, lors de la reprise du cours sur la langue :

\begin{abstract}
Foule réunie sur une place de marché ; de quelle manière la langue est-elle présente dans cette foule? Sous forme d'un dépôt <existant dans cerveau $>$ de chacune des personnes composant la foule $<$ comme un dictionnaire dont tous les exemplaires seraient répartis entre ces personnes>. Cette chose bien qu'intérieure à chaque individu est en même temps bien collectif, qui est placé hors de la volonté de l'individu. (10 : p. 236).
\end{abstract}

La définition saussurienne de la langue comme «articulation de la pensée dans la matière phonique » est bien connue par le Cours de linguistique générale [12], où elle apparaît dans le premier paragraphe du quatrième chapitre de la deuxième partie, dont nous reprenons ici le titre ${ }^{21}$. Elle provient des deuxième et troisième cours ${ }^{22}$, où Saussure s'attache à définir ce qu'il appelle, dans le troisième cours, le " fait linguistique », le « fait $<$ en quelque sorte> mystérieux que la pensée-son implique des divisions qui sont les unités finales de la linguistique » $(9:$ p. 21$)$ et qui consiste à «créer un milieu intermédiaire de telle $<$ nature $>$ que le compromis entre la pensée et le son aboutit d'une façon inévitable à des unités $<$ particulières $>$ » $(9:$ p. 21$)$. Avant la langue, il n’y a ni idées ni unités phoniques préexistantes, mais « [c]'est entre deux que le fait linguistique se passe » (10:p. 285), fait linguistique qui «donnera naissance à des valeurs qui elles <pour la première fois> seront déterminées, mais qui n'en resteront pas moins des valeurs, avec le sens qu'on peut attacher à ce mot » $(10:$ p. 285). Il apparaît, à la lecture de ces différentes propositions, que cette définition de la langue s'inscrit dans une problématique étiologique. Le " fait linguistique », ainsi défini, vaut étiologie du signe et des idiomes en tant que systèmes de signes; la langue saussurienne n'est pas un "système de signes", au sens où le définissent Damourette et Pichon ${ }^{23}$, mais, comme y insiste Saussure dans ce passage du troisième cours, un système de valeurs, « avec le sens que l'on peut attacher à ce mot », c'est-à-dire d' « articuli » (9: p. 22), qui ne sont pas des signes, au sens commun de la combinaison d'un signifié et d'un signifiant, mais des entités dont l'existence se confond avec la délimitation, délimitation pour sa part inséparable d'un «accouplement » (9: p. 22) de la pensée avec la matière phonique : ce sont de «petits membres dans lesquels la pensée prend conscience $<$ (valeur ? B. $)>$ par un son $»(9:$ p. 22). Dans cette perspective, la langue apparait, non comme un objet - structure, grammaire ou système de signes -, mais comme un fonctionnement - cette délimitation-combinaison, qui se fait toujours à nouveau, et dont le renouvellement permanent constitue proprement la langue - dont son, sens et idiomes sont les effets.

La définition saussurienne de la langue rompt ainsi avec la conception plurimillénaire du signe comme combinaison d'un signifiant et d'un signifié, d'une forme et d'une 
signification - peu importe, à cet égard, la terminologie retenue. Le signe, au sens saussurien, n'est en effet pas analysable en un signifiant et un signifié, mais il n'existe comme tel que dans l'instant de sa constitution, et s'oppose ainsi à la « figure vocale » qu'il redevient chaque fois, et qui n'est «signifiant» que comme effet de langue, c'est-à-dire d'un point de vue idiomologique. Citons notamment ce passage bien connu de «De l'essence double du langage » [dans 7]:

Le dualisme profond qui partage le langage ne réside pas dans le dualisme du son et de l'idée, du phénomène vocal et du phénomène mental ; c'est là la façon facile et pernicieuse de le concevoir. Ce dualisme réside dans la dualité du phénomène vocal COMME TEL, et du phénomène vocal COMME SIGNE - du fait physique, (objectif) et du fait physico-mental (subjectif), nullement du fait «physique » du son par opposition au fait «mental» de la signification. Il y a un premier domaine, intérieur, psychique, où existe le signe autant que la signification, l'un indissolublement lié à l'autre ; il y en a un second, extérieur, où n'existe plus que le «signe»; mais à cet instant le signe réduit à une succession d'ondes sonores ne mérite pour nous que le nom de figure vocale. $(7:$ p. $20-21)$.

La linguistique de Damourette et Pichon se situe pour sa part dans la problématique antérieure. En témoigne, notamment, la réfutation du principe de l'arbitraire du signe, que Saussure situe au fondement du concept de valeur - il désigne la nécessité d'une étiologie du signe -, mais auquel Damourette et Pichon opposent la consubstantialité du signifiant et du signifié - au sens commun -, raisonnant ainsi en termes de types de rapports son/sens, donc dans le cadre de la définition traditionnelle du signe. Pichon écrit ainsi notamment en 1937 dans « La linguistique en France. Problèmes et méthodes » [13] :

Il nous semble que la façon dont M. Damourette et moi voyons la question serre de beaucoup plus près la réalité vivante. J'en rappelle l'essentiel : le signifiant (mot) et le signifié (idée) ne sont pas liés entre eux par un rapport causal, mais par un véritable rapport existentiel, si j'ose ainsi parler; l'idée (qui est ici le signifié) est, avons-nous écrit (EGLF, § 58), profondément incluse dans le mot; elle lui est intérieure, elle est en quelque sorte l'âme dont il est le corps. (13: p. 29-30).

Il s'agit bien en effet, ici, de « rapport » - « causal » ou « existentiel » - entre signifiant et signifié, au lieu que l'arbitraire saussurien est au fondement de la relativité des valeurs, c'est-à-dire, aussi bien, de leur négativité, et ainsi de la définition des signes comme articuli $^{24}$. L'équivalence posée entre signifié et idée d'une part, signifiant et mot d'autre part, dit d'ailleurs assez le caractère commun de ces notions dans la représentation de Damourette et Pichon. Si ces derniers reprennent ces termes saussuriens, c'est dans le cadre de la conception traditionnelle du signe comme un aliquid quod stat pro aliquo, qui apparaît par exemple dans ce passage de Des mots à la pensée. Essai de grammaire de la langue française, où l'on notera en particulier la notion de représentation :

Que les idées humaines n'aient de valeur que relative et par rapport aux hommes, ou qu'au contraire elles leur apportent du monde une connaissance en soi et douée de valeur objective absolue, c'est une question métaphysique qui dépasse celle que nous traitons ici ; mais il est certain que les mots ne représentent que les idées; qu'ils sont, comme il a été dit au $\S 58$, le corps de celles-ci, et qu'il y a par conséquent une contradiction logique à croire que chaque mot ne soit qu'arbitrairement et fortuitement le représentant de telle idée plutôt que de telle autre. (1, tome I : p. 96). 
Cette notion de représentation se double cependant, comme il apparaît dans ces deux passages, de celle de consubstantialité, avec la métaphore de l'âme et du corps. Le rapport son/sens, chez Damourette et Pichon, se redouble en effet en construction d'une " penséelangage » (par exemple $13:$ p. 35 et $38 ; 1$, tome I : p. 146). Le «signifié » devient ainsi, à l'échelle de l'idiome, un «système de pensée » (par exemple 1, tome I : p. $240 ; 13$ : p. 36) ou «état mental synchronique » (1, tome I : p. 382). Le propos des deux linguistes semble ici rencontrer celui de Saussure. On lit ainsi par exemple dans l'article de Pichon de 1937 :

\begin{abstract}
Saussure reconnaît lui-même (CLG, p. 99) qu'il n'y a, humainement, pas de concepts généraux préexistant aux mots. C'est grâce à l'avènement de la pensée lingui-spéculative que peuvent se constituer chez l'homme les idées abstraites, outils mentaux plus souples que les images unisensorielles ou les conglomérats d'images pluri-sensoriels de la pensée sensu-actorielle.

Dès lors, si l'on pose le problème comme le pose Saussure, c'est-àdire si c'est à l'idée qu'on fait jouer le rôle de signifié, le rapport entre elle et le signifiant n'est pas contingent, n'est pas arbitraire. Il est nécessaire, puisqu'il est constitutif de l'idée même. Le mot est le corps même de l'idée. L'idée de bøuf n'existe pas indépendamment de la suite phonétique [b-oè-f]. $(13:$ p. 26$)$.
\end{abstract}

À cet endroit du Cours de linguistique générale, il est écrit que la conception de la langue comme une nomenclature est «critiquable à bien des égards» (12: p. 97), notamment parce qu'elle «suppose des idées toutes faites préexistant aux mots (sur ce point, voir plus loin, p. $155 »(12:$ p. 97). La page 155 appartient au premier paragraphe du quatrième chapitre de la deuxième partie du Cours de linguistique générale mentionné plus haut, où on lit de fait :

\begin{abstract}
Psychologiquement, abstraction faite de son expression par les mots, notre pensée n'est qu'une masse amorphe et indistincte. Philosophes et linguistes se sont toujours accordés à reconnaître que, sans le secours des signes, nous serions incapables de distinguer deux idées d'une façon claire et constante. Prise en elle-même, la pensée est comme une nébuleuse où rien n'est nécessairement délimité. Il n'y a pas d'idées préétablies, et rien n'est distinct avant l'apparition de la langue. $(12:$ p. 155$)$.
\end{abstract}

Le propos de Pichon apparaît déjà significativement différent de celui du Cours de linguistique générale : il est question, dans le passage cité, non de " pensée » et d' "idées » comme dans ce dernier, mais de «concepts généraux » et d' «idées abstraites ». De fait, la «pensée lingui-spéculative» n'est pas pensée articulée dans le langage, au sens de la définition saussurienne de la langue, c'est-à-dire au sens d'un effet de langue, langue dont, dès lors, la pensée est distincte, puisqu'elle est seulement un élément entrant en jeu dans le fonctionnement de celle-ci, mais un type de pensée, un outil dont s'est progressivement dotée la «puissance cognitive» (14: p. 37) humaine, dès lors, effectivement, "penséelangage », objet à la fois linguistique et psychique - comme il apparaîtra ci-dessous, on pourrait dire, et même on devrait dire, pour mieux établir la différence avec l'élaboration saussurienne, "psychologique ». À l'étiologie saussurienne se substitue ainsi une genèse, c'est-à-dire une reconstruction, à partir du langage défini comme "pensée-langage », de manière éminemment empirique, de la constitution progressive du langage caractérisé comme «pensée lingui-spéculative» ou, comme il est apparu plus haut, «partie proprement humaine de la pensée des hommes » $\left(1\right.$, tome I : p. 16) ${ }^{25}$. Cet empirisme apparaît de manière très nette dans l'assomption du point de vue du locuteur, lisible par exemple dans ce passage de « La linguistique en France. Problèmes et méthodes »:

Pourquoi et comment la suite phonétique [b-oè-f] a-t-elle pris la valeur que nous lui connaissons, c'est une question diachronique. Dans la 
psychologie linguistique d'un sujet parlant, le complexe idée-mot bruf est constitué; l'idée signifiée et le mot signifiant y sont coalescents l'un à l'autre en une adéquation parfaite et sans arbitraire. $(13:$ p. 28$)$.

Le point de vue du locuteur est en effet un point de vue de première venue, celui même qui préside à la définition traditionnelle du signe, que, comme nous l'avons écrit ci-dessus, la définition de la langue comme «pensée-langage » ne fait que redoubler : la distinction entre «pensée sensu-actorielle » et "pensée lingui-spéculative » est une distinction entre deux types de pensée, la seconde étant ensuite définie, en second lieu, et sur le fond de la définition traditionnelle du signe, par la coalescence entre mot et idée, son émergence se confondant dès lors avec celle du langage. Citons notamment ces propositions de Pichon dans « Comment la pensée prend corps » (1938) [14] :

Or il y a une distinction essentielle à établir, dans la pensée humaine, entre deux modes qui fonctionnent concurremment: d'une part, un mode ancien, que nous avons toute raison de croire représenté aussi chez les animaux, c'est la pensée sensu-actorielle : le sujet perçoit des objets, il a des percepts; ou bien, dans son esprit, il évoque des images mentales ou typomes: images sensorielles de ces objets, images motrices des actes qu'il pourra exécuter envers eux. Ces percepts et ces typomes constituent un premier mode de pensée, lequel ne permet que des cogitations particulières; tout au plus peut-on y glisser du particulier au particulier, ce que ceux qui étudient la psychologie des enfants ont appelé la transduction; mais, dans ce mode de fonctionnement mental, il n'y a pas de vrai jugement possible.

Telle est la pensée sensu-actorielle, telle qu'elle existerait si nous ôtions de notre psychisme tout ce qui n'est pas le langage.

$\mathrm{Au}$ moment où le langage intervient, est créé de ce fait un mode entièrement nouveau de pensée: la pensée lingui-spéculative: l'accession à l'abstrait, au général est rendue possible par la création d'outils ad hoc, que nous appellerons les idées proprement dites, corporées dans des mots.

Le langage, dans l'état où il est parvenu actuellement, rend possibles les jugements véritables, et permet, par conséquent, les plus hautes spéculations de l'esprit. (14:p. 37-38).

Il faut ainsi souligner le caractère purement idiomologique de la «pensée-langage » de Damourette et Pichon, qualificatif que l'on peut entendre en deux sens distincts, qu'il importe de ne pas confondre : il s'agit, certes, de description d'un idiome, objet légitime de la linguistique - qui se fait alors «idiomologie»-, mais surtout, cette conception de l'idiome est présentée comme une conception linguistique, alors qu'elle a été élaborée sans rupture avec l'empirisme: il s'agit dès lors d'idiomologie indûment faite linguistique. L'objet ainsi construit, "pensée-langage » ou « pensée lingui-spéculative » n'a donc rien de linguistique : il s'agit d'un objet valorisé, et non théorisé, et c'est là l'enjeu de toute linguistique "psychologique». Notons que cet objet, en dépit des allégations de Damourette et Pichon, n'a rien non plus de psychanalytique. Le langage, ainsi conçu, est certes défini comme un système de pensée inconscient, dont l'étude permet dès lors d'accéder à l' « inconscient collectif» de la communauté des locuteurs concernés. On lit ainsi dans « La grammaire en tant que mode d'exploration de l'inconscient » (1925) [16] :

Un parler est donc essentiellement un système de pensée, et un système inconscient pour la plus grande part.

Amener à la lumière les ressorts inconscients d'un système taxiématique, ce sera donc un des moyens les plus efficaces pour 
pénétrer le fonctionnement de l'inconscient dans une collectivité nationale donnée. (16: p. 240).

Citons également un peu plus loin dans le même article :

En somme, si par d'autres méthodes on peut espérer connaître ce qu'il y a d'individuel dans l'inconscient, la grammaire, seule, parait pouvoir nous éclairer sur cet inconscient national collectif qui s'appelle le génie d'un peuple. Et même peut-être, comme en réalité il n'y a pas deux personnes dont le parler soit rigoureusement identique, peut-on espérer que le jour où sera suffisamment avancé ce qu'on pourrait appeler la grammaire nationale moyenne, les particularités spéciales observées chez tel ou tel individu pourront contribuer à éclairer son inconscient individuel. De sorte que les écrivains arriveraient à nous livrer, par leur style même, l'histoire de leur âme. (16: p. 256-257).

Il ne suffit cependant pas de parler d'inconscient pour se situer dans la problématique psychanalytique, problématique dont il est clair, à la lecture, par exemple, de ce passage de « La linguistique en France. Problèmes et méthodes », que Pichon est très éloigné :

Le second point qui a égaré Saussure n'est pas une doctrine positive mais une carence. Lui dont le fils (Raymond de Saussure) devait être un des pionniers de la psychanalyse en Suisse romande, il ignorait les étages du psychisme quant à la clarté de la conscience. Il fallait que M. Damourette et moi-même eussions, au pays de Pierre Janet, nettement la notion d'une couche subconsciente capable de régler bon nombre de réalisations actuelles pour que nous en arrivions à situer dans cette couche l'essentiel du système d'un idiome, à vouloir aller l'y déterrer et à considérer les aberrances et même certains lapsus comme de précieuses émergences des filons à retrouver. $(13:$ p. 36).

La psychanalyse de Freud se pose en effet en rupture avec la psychologie de Janet et, par ailleurs, l'inconscient freudien n'a rien d'une "couche subconsciente ». Enfin, et surtout, si la psychanalyse et la linguistique ont toutes deux affaire au langage, elles ont pourtant deux objets radicalement distincts : respectivement, le locuteur et la langue.

Ni linguistique, ni psychanalytique, l'objet construit par Damourette et Pichon est ainsi avant tout imaginaire, d'où l'immixtion de considérations très éloignées de la réflexion scientifique : revendications nationalistes, propositions maurrassiennes, etc., dont Valelia Muni Toke [2] souligne avec raison qu'elles sont des éléments à part entière de l'élaboration théorique des deux linguistes français. Apparaît de nouveau ici, la manière dont se conjuguent et se renforcent idéologie et idéologie scientifique.

\section{Conclusion}

Il nous faut cependant insister, pour conclure, sur le fait que s'il nous paraît si important de mettre en évidence l'obstacle épistémologique de l'idiome, dont apparaît ici le caractère déterminant dans l'élaboration de Damourette et Pichon, c'est en raison de l'importance des enjeux de celui-ci pour les sciences du langage actuelles. Cet obstacle épistémologique est en effet protéiforme, et les formes qu'il prend ont pour point commun un empirisme descriptif, lieu de toutes les idéologies, scientifiques ou non. Si le psychologisme nationaliste de Damourette et Pichon est sans conteste daté, on peut en revanche penser que le psychologisme se maintient aujourd'hui sous une forme que l'on pourrait dire « inversée » : celle de l'organicisme, tout aussi idéologique. Dans son compte rendu de la publication du troisième cours par Komatsu [15], Martinet écrit, à propos de la définition saussurienne de la langue, où il voit une « abstraction universaliste » $(15:$ p. 144) : 
Cette dernière trace de la conviction philosophique d'une opposition entre l'esprit et la matière, l' «âme » et le « corps », se voit rejetée aux vieilles lunes et l'essentiel de ce troisième cours tel qu'il transparaît dans le relevé de Constantin est parfaitement caduc. $(15:$ p. 144)

«Conviction philosophique », voire religieuse, la dualité âme/corps n'en correspond pas moins à une certaine réalité, que l'on pourrait caractériser comme celle de l'hétérogénéité du langage et de l'animal, ou de la discontinuité séparant logique du langage et logique du vivant. Il faut cependant constater que cette dualité demeure aujourd'hui impensable, n'étant que redoublée sous la forme de la dualité entre "psychologisme» et «organicisme", et, peut-être, dans la dualité entre "sciences humaines » et « sciences de la nature », que l'idéologie scientifique de la " formalisation » en sciences humaines (dont le parangon est le structuralisme, par exemple martinettien), aussi bien que le scientisme des sciences biologiques du langage et de la psychiatrie, s'attachent chacun pour leur part à creuser, au lieu qu'il s'agirait peut-être de la redéfinir.

\section{Références}

1. Damourette, J. \& Pichon É. (1911-1927). Des mots à la pensée. Essai de grammaire de la langue française. Paris : D'Artrey.

2. Muni Toke, V. (2013). La grammaire nationale selon Damourette et Pichon (1911-1939). L'invention du locuteur. Lyon : ENS éditions.

3. Guillaume, G. (1989). Leçons de linguistique de Gustave Guillaume 1946-1947 C. Grammaire particulière du français et grammaire générale II. Québec : Presses de l'Université Laval/Lille : Presses universitaires de Lille.

4. Canguilhem, G. (2000). Idéologie et rationalité dans l'histoire des sciences de la vie Paris : Vrin.

5. Paris, G. (1888). Les parlers de France. Lecture faite à la réunion des sociétés savantes le samedi 26 mai 1888. Revue des patois gallo-romans, II, p. 161-175.

6. Schmitt, J. (1872). Die Verwandtschaftsverhältnisse der Indogermanischen Sprachen. Weimar: Hermann Böhlau.

7. Saussure, F. (de). (2002). Écrits de linguistique générale. Paris : Gallimard ${ }^{26}$.

8. Saussure, F. (de). (1996). Premier cours de linguistique générale (1907), d'après les cahiers d'Albert Riedlinger. Oxford, New York, Seoul, Tokyo : Pergamon.

9. Saussure, F. (de). (1997). Deuxième cours de linguistique générale (1908-1909), d'après les cahiers d'Albert Riedlinger et Charles Patois. Oxford, New York, Seoul, Tokyo : Pergamon.

10. Saussure, F. (de) \& Constantin, É. (2005). Ferdinand de Saussure : Notes préparatoires pour le cours de linguistique générale 1910-1911, Emile Constantin : Linguistique générale. Cours de M. le professeur de Saussure 1910-1911. Cahiers Ferdinand de Saussure, 58, p. 83-289.

11. Saussure, F. (de). (1986). Le leggende germaniche. Este : Zielo editore.

12. Saussure, F. (de). (1995). Cours de linguistique générale. Paris : Payot.

13. Pichon, É. (1937). La linguistique en France. Problèmes et méthodes. Journal de psychologie normale et pathologique, XXXIV, p. 25-48

14. Pichon, É. (1938). Comment la pensée prend corps. A l'aise dans la civilisation, III. Revue française de psychanalyse, $X-1$, p. 34-49.

15. Martinet, A. (1995). [Compte rendu de] Komatsu, Eisuke et Harris, Roy, dir., Troisième cours de linguistique générale (1910-1911) de Ferdinand de Saussure, d'après les notes d'Émile Constantin, et version anglaise : Saussure's Third Course of Lectures on General Linguistics (1910-1911), Oxford, Tokyo, Pergamon, 1993, XXIV + 174 pages. La Linguistique, XXXI-1, p. $143-145$.

16. Damourette, J. \& Pichon É. (1925). La grammaire en tant que mode d'exploration de l'inconscient. L'Évolution psychiatrique, 1, p. 237-257.

\footnotetext{
${ }^{1}$ Voir par exemple 1, tome I : p. 12.

${ }^{2}$ Ces deux expressions ne sont bien entendu pas équivalentes ; la première, outre qu'elle reprend les termes mêmes qu'utilisent Damourette et Pichon, indique une problématique idiomologique ; elle est
} 
donc, comme il apparaîtra dans ce qui suit, bien plus appropriée que la seconde. Voir cependant 2 : p. 21 .

${ }^{3}$ C'est-à-dire le système des «idées directrices servant au classement sommaire des idées-matériaux [les sémièmes, qui sont « un matériel idées pouvant s'accroître indéfiniment » $(1$, tome I : p. 11), des « idées n'ayant pas de valeur spéciale dans la texture du langage » $(1$, tome I : p. 12)] et à leur mise en œuvre dans le discours », et constituant ainsi la «charpente » (1, tome I : p. 12) du langage.

${ }^{4} \mathrm{Il}$ y a longtemps, en effet, à présent, que la notion de race a perdu tout droit de cité en biologie.

${ }^{5}$ On lisait déjà plus haut, introduisant à ce développement relatif aux rapports entre race et langue : «L'homme étant essentiellement l'animal qui parle, il est naturel de prendre la langue comme critère de classification, zoologique pourrait-on dire, des différentes variétés du genre humain. Mais il nous faut analyser de près maintenant à quoi correspond au point de vue des groupements collectifs la notion de langue. » $(1$, tome $\mathrm{I}: \mathrm{p}$. 17).

${ }^{6}$ Voir 1 , tome I : p. 17-18.

${ }^{7}$ Voir 1 , tome I : p. 24 sqq.

${ }^{8}$ Voir 1 , tome I : p. 18 sqq.

${ }^{9}$ Voir 1, tome I : p. 20. S'opposent alors des « langues à patois », comme le français, et des «langues à dialectes », comme l'allemand. Voir de même 1, tome I : p. 20.

${ }^{10}$ Voir 1 , tome I : p. 23.

${ }^{11}$ Voir 1 , tome I : p. 39.

${ }^{12}$ Voir 1 , tome I : p. 42 sqq.

${ }^{13}$ Voir également 1, tome I : p. 42.

${ }^{14}$ Voir $7:$ p. 170-172. Ces affirmations sont reprises dans les trois cours de linguistique générale. Voir $8:$ p. $44,9:$ p. $99-100$ et $10:$ p. 100 puis $117-126$.

${ }^{15}$ Voir $8:$ p. $107-111,9:$ p. $97-104$ et $10:$ p. 93-143. Voir aussi $7:$ p. 169.

${ }^{16}$ Voir auparavant $10:$ p. 95.

${ }^{17}$ Selon une expression de notes préparatoires de Saussure. Voir 7 : p. 309-310.

${ }^{18}$ Voir notamment, à cet égard, 10 : p. 139-140.

${ }^{19}$ Voir 10 : p. 128-139.

${ }^{20}$ Voir également notamment les notes relatives aux légendes germaniques [11].

${ }^{21}$ Voir 12 : p. 155-157.

${ }^{22}$ Voir $9:$ p. $21-22$ et $10:$ p. 285.

23 «Pour cet auteur [Saussure], la langue doit être envisagée objectivement comme un système de signes ayant et une valeur sociale et une fixité relative qui le rendent scientifiquement étudiable. » (1, tome I : p. 10).

${ }^{24}$ Rappelons notamment ce passage du troisième cours - significativement inversé dans le Cours de linguistique générale: "Non seulement ces deux domaines entre lesquels se passe le fait linguistique sont amorphes, <mais le choix du lien entre les deux> ce mariage <entre les deux> qui créera la valeur est parfaitement arbitraire. Sans cela, les valeurs seraient dans une certaine mesure absolues. $<$ Si ce n'était pas arbitraire, il y aurait à restreindre cette idée de valeur, il y aurait un élément absolu $>$

Mais puisque ce contrat est parfaitement arbitraire, les valeurs seront parfaitement relatives. » (10 : p. 285-286).

${ }^{25}$ Voir 1, tome I : p. 67-76. 
${ }^{26}$ Signalons que toutes nos citations ont été corrigées sur les manuscrits. Le texte peut donc différer de celui des Écrits de linguistique générale auxquels nous nous référons. 